\title{
MULTI-SCALE THERMAL-HYDRAULIC ANALYSIS OF PWRS USING THE CUPID CODE
}

\author{
HAN YOUNG YOON ${ }^{1}$, HYOUNG KYU CHO ${ }^{1}$, JAE RYONG LEE ${ }^{1}$, IK KYU PARK ${ }^{1}$, and JAE JUN JEONG ${ }^{2 *}$ \\ ${ }^{1}$ Korea Atomic Energy Research Institute \\ 1045 Daeduk-daero, Daejeon, Korea \\ ${ }^{2}$ Pusan National University \\ 30, Jangjeon-dong, Keumjeoung-gu, Busan, Korea, 609-735 \\ ${ }^{*}$ Corresponding author. E-mail : jjjeong@pusan.ac.kr
}

Received November 07, 2012

KAERI has developed a two-phase CFD code, CUPID, for a refined calculation of transient two-phase flows related to nuclear reactor thermal hydraulics, and its numerical models have been verified in previous studies. In this paper, the CUPID code is validated against experiments on the downcomer boiling and moderator flow in a Calandria vessel. Physical models relevant to the validation are discussed. Thereafter, multi-scale thermal hydraulic analyses using the CUPID code are introduced. At first, a component-scale calculation for the passive condensate cooling tank (PCCT) of the PASCAL experiment is linked to the CFD-scale calculation for local boiling heat transfer outside the heat exchanger tube. Next, the Rossendorf coolant mixing (ROCOM) test is analyzed by using the CUPID code, which is implicitly coupled with a systemscale code, MARS.

KEYWORDS : Thermal-hydraulics, Multi-scale Analysis, Two-phase Flow, CUPID

\section{INTRODUCTION}

Recent advances in computational fluid dynamics (CFD) codes along with high performance computing hardware made the codes more useful in many engineering applications. For instance, single-phase CFD codes have long been used to predict the fluid flow in fuel channels of a pressurized water reactor (PWR) [1,2]. Considering the current affordable number of computation mesh is a little below one billion, one fuel assembly of a PWR is now being simulated using a single-phase RANS model. The whole core of a PWR, which requires hundreds of billions of meshes for realistic simulation, is expected to be analyzed using CFD over the next decade. A two-phase flow also appears in many PWR transient and accident analyses. However, the two-phase CFD simulation has been challenging up to now mainly because of insufficient physical models for a two-phase flow in the CFD scale. Most of the current physical models for a two-phase flow have been developed for system codes, such as MARS [3], REALP5 [4] and CATHARE [5], which adopt onedimensional governing equations. In addition, the numerical solution methods of current CFD codes are sometimes not efficient in dealing with two-phase flows because interface mass, momentum and energy transfer terms are usually treated in an explicit manner as non-linear source terms in the governing equations.
A two-phase CFD code, CUPID [6-9], has been developed at KAERI for the analysis of transient two-phase flows in nuclear reactor components. The CUPID code employs a two-fluid three-field model. The governing equations are discretized using the finite volume method (FVM) with unstructured grids, and solved using a semiimplicit numerical method. This is useful for the analysis of transient two-phase flows where the numerical solution hardly converges using the SIMPLE-based implicit method. The numerical method of the CUPID code has been verified against a set of single- and two-phase test problems. Validation of the CUPID code has also been carried out using experiment data.

In this paper, two validation cases are introduced. The downcomer boiling (DOBO) [10] experiment performed at KAERI is analyzed to validate the boiling heat transfer model in open media. Then a test from STERN laboratory [11] simulating the moderator flow in a Calandria vessel is assessed using the porous media model of the CUPID code.

For more practical engineering applications, the concept of "multi-scale" analysis has been proposed $[12,13]$ by adopting the combined use of different scale computational tools, such as system and CFD codes, since direct use of two-phase CFD codes for nuclear reactor system analysis requires huge computational cost. The multi-scale analysis 
method can be either an "explicit (weak)-coupled" or an "implicit (strong)-coupled" one. In an explicitly-coupled method, the simulation results obtained from a larger lengthscale mesh are used as boundary conditions for a refined calculation with a smaller length-scale mesh. This method is applied to the analysis of boiling heat transfer outside the heat exchanger tube submerged in the PCCT [14]. The liquid temperature, velocity, and pressure obtained from the PCCS calculation are used as boundary conditions for the analysis of boiling heat transfer of the heat exchanger tube in open media.

On the other hand, in an implicitly-coupled method, a smaller length-scale code is coupled with a larger lengthscale code at the source level. In this work, a CFD scale code, CUPID, has been coupled with a system-scale code, MARS, implicitly by solving the pressure equations of the two codes simultaneously. This has an advantage over the explicitly-coupled method for the analysis of rapid two-phase flow transients. One of the ROCOM tests [15] has been successfully analyzed using the coupled code, MARS-CUPID, where the pressure vessel is calculated by the CUPID code and the other components such as hot and cold legs are simulated by the MARS code.

\section{MATHEMATICAL MODELS FOR TWO-PHASE FLOWS}

A transient two-fluid, three-field model [16, 17] is adopted in the CUPID code to model the thermal-hydraulics of PWRs, where the two fluids are vapor and liquid, and the three fields are vapor, continuous liquid and droplet fields. A major application of the CUPID code is the analysis of transient two-phase flow with a resolution ranging from the CFD scale to the component scale. Porosity and permeability of a computing cell are modeled for a component scale application and different physical models are applied depending on the computation scale.

\subsection{Governing Equations}

Separate conservation equations of mass, momentum, and energy are established for the three fields. The mass conservation equation for the $\mathrm{k}$-field is:

where

$$
\frac{\partial}{\partial t}\left(\alpha_{k} \rho_{k}\right)+\nabla \cdot\left(\alpha_{k} \rho_{k} \vec{u}_{k}\right)=\Omega_{k}
$$

$$
\begin{aligned}
& \Omega_{v}=\Gamma_{v}, \\
& \Omega_{l}=-(1-\eta) \Gamma_{v}-S_{E}+S_{D E}, \\
& \Omega_{d}=-\eta \Gamma_{v}+S_{E}-S_{D E}, \\
& \eta=\alpha_{d} /\left(\alpha_{l}+\alpha_{d}\right) .
\end{aligned}
$$

Non-condensable gases are considered to be present in the vapor field. Velocity and temperature of the noncondensable gas are assumed to be the same as those of the vapor. Thus, only the mass conservation of the non- condensable gas is included in the governing equation:

$$
\frac{\partial}{\partial t}\left(\alpha_{v} \rho_{v} X_{n}\right)+\nabla \cdot\left(\alpha_{v} \rho_{v} X_{n} \vec{u}_{v}\right)=0
$$

where the non-condensable gas quality $X_{n}$ is defined as the ratio of the non-condensable gas mass to the total mixture mass of gas and vapor.

The conservation equation of momentum for the kfield is:

$$
\begin{aligned}
\frac{\partial}{\partial t}\left(\alpha_{k} \rho_{k} \vec{u}_{k}\right)+ & \nabla \cdot\left(\alpha_{k} \rho_{k} \vec{u}_{k} \vec{u}_{k}\right)= \\
& -\alpha_{k} \nabla P+\nabla \cdot\left[\alpha_{k}\left(\mu_{l}+\mu_{t}\right) \nabla \vec{u}_{k}\right]+\alpha_{k} \rho_{k} \vec{g} \\
& +\mathbf{M}_{k}^{\text {mass }}+\mathbf{M}_{k}^{\text {drag }}+\mathbf{M}_{k}^{\text {ndrag }}+\mathbf{M}_{k}^{V M}
\end{aligned}
$$

where the last four terms are the interfacial momentum transfer terms due to phase change, drag force, non-drag forces, and virtual mass force, respectively. For the turbulent viscosity $\mu_{t}$ in Eq. (3), both the mixing length model and the k-e model are implemented in the CUPID code.

For the energy conservation equation, a thermal equilibrium is assumed between the continuous liquid and droplet fields, i.e., $T_{d}=T_{l}$ and $\rho_{d}=\rho_{l}$. Thus, only two energy equations are used: one for the vapor field and the other for the combined liquid field. They are given as:

$$
\begin{gathered}
\frac{\partial\left(\alpha_{g} \rho_{g} e_{g}\right)}{\partial t}+\nabla \cdot\left(\alpha_{g} \rho_{g} e_{g} \vec{u}_{g}\right)= \\
-P \frac{\partial \alpha_{g}}{\partial t}-P \nabla \cdot\left(\alpha_{g} \vec{u}_{g}\right)+\dddot{q}_{g}+\nabla\left(\alpha_{g} \vec{q}_{g}\right), \\
+\frac{P_{s}}{P} H_{i g}\left[T^{s}\left(P_{s}\right)-T_{g}\right]+\Gamma_{v} h_{g}^{*}-\left(\frac{P-P_{s}}{P}\right) H_{g f}\left(T_{g}-T_{l}\right) \\
\frac{\partial\left\{\left(\alpha_{l}+\alpha_{d}\right) \rho_{l} e_{l}\right\}}{\partial t}+\nabla \cdot\left(\alpha_{l} \rho_{l} e_{l} \vec{u}_{l}+\alpha_{d} \rho_{d} e_{d} \vec{u}_{d}\right)= \\
-P \frac{\partial\left(\alpha_{l}+\alpha_{d}\right)}{\partial t}-P \nabla \cdot\left(\alpha_{l} \vec{u}_{l}+\alpha_{d} \vec{u}_{d}\right) \\
+\dddot{q}_{l}+\nabla \cdot\left(\alpha_{l} \vec{q}_{l}+\alpha_{d} \vec{q}_{d}\right)+H_{i f}\left[T^{s}\left(P_{s}\right)-T_{l}\right] \\
-\Gamma_{v} h_{f}^{*}+\left(\frac{P-P_{s}}{P}\right) H_{g f}\left(T_{g}-T_{l}\right)
\end{gathered}
$$

where $\vec{q}_{k}$ is the conduction heat flux given by $\vec{q}_{k}=\kappa_{k} \nabla T_{k}$. Note that energy dissipation due to shear stress force is not considered. The interfacial heat transfer coefficient $H_{i k}$ is explained in Section 2.2.

The density and temperature of each phase are expressed as functions of the independent variables, the pressure and internal energy.

$$
\begin{aligned}
& \rho_{l}=\rho_{l}\left(P, e_{l}\right), \\
& T_{l}=T_{l}\left(P, e_{l}\right) .
\end{aligned}
$$

The properties for the gaseous phase are calculated assuming a modified Gibbs-Dalton mixture of vapor and an ideal non-condensable gas:

$$
\begin{gathered}
\rho_{v}=\rho_{v}\left(P, e_{v}, X_{n}\right), \\
T_{v}=T_{v}\left(P, e_{v}, X_{n}\right),
\end{gathered}
$$




$$
P_{s}=P_{s}\left(P, e_{v}, X_{n}\right) .
$$

The saturation temperature is represented as a function of the pressure:

$$
T_{\text {sat }}=T_{\text {sat }}\left(P_{s}\right) .
$$

\subsection{Physical Models}

For a mathematical closure of the governing equations, constitutive relations for the interfacial transfer terms are necessary depending on the flow patterns and their interphase shape. In CUPID, an inter-phase topology map proposed by Tentner [18] is used. Fig. 1 shows the concept of the inter-phase topology map where three regions are defined for bubbly, mist, and stratified flow regimes. Transition criteria for the three flow regimes are determined by the void fraction and the gradient of void fraction, which are defined as:

where

$$
\gamma=0.5 \cdot \vec{\delta} \cdot \nabla \alpha
$$

$$
\vec{\delta}=\frac{V_{i}}{\sum_{f}\left|S_{f, x}\right| / N_{f}} \vec{i}+\frac{V_{i}}{\sum_{f}\left|S_{f, y}\right| / N_{f}} \vec{j}+\frac{V_{i}}{\sum_{f}\left|S_{f, z}\right| / N_{f}} \vec{k} .
$$

Specific values of the transition criteria are:

$$
\alpha_{g, b c}=0.3, \alpha_{g, c m}=0.9, \gamma_{1}=0.2, \gamma_{2}=0.45 \text {. }
$$

Flow regimes among the three independent regions are regarded as transition regions, where the interfacial terms are linearly interpolated from the values of the bubbly, mist, and stratified regions.

Once the flow regime is determined, the interfacial area is calculated. An interfacial area concentration (IAC) model or the interfacial area transport (IAT) equation is used in the CUPID code. In the IAC model, the interfacial area for the bubbly and mist flow regime is given by

$$
A_{i}=\frac{6 \alpha_{k}}{D_{k}}
$$

where $D_{k}$ is the bubble or droplet diameter of the dispersed phase. Hibiki [19] and Kataoka[20] correlations are ad-

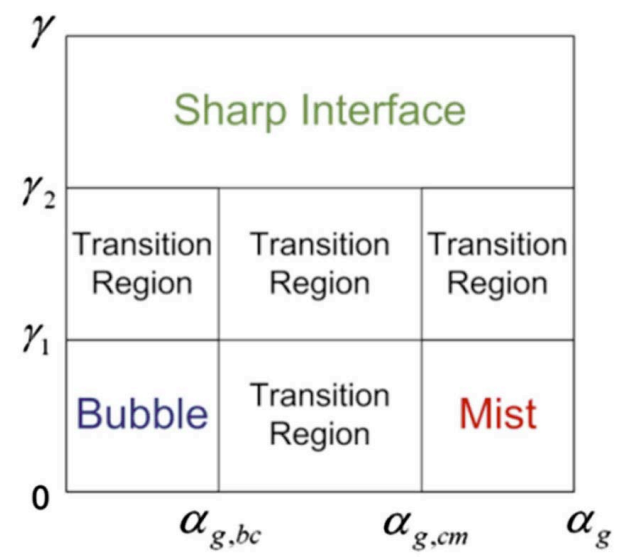

Fig. 1. Inter-phase Topology Map opted for the bubble and drop diameters, respectively. For the stratified flow regime [21], the interfacial area is calculated by

$$
A_{i}=\left|\nabla \alpha_{g}\right|
$$

The interfacial momentum transfer term includes the drag, lift, wall lubrication, turbulent dispersion and virtual mass forces. The interfacial drag force for the bubbly and mist flow regimes is expressed as

$$
\vec{F}_{g l}=\frac{1}{8} A_{i} \rho_{c} C_{D}\left|\vec{u}_{g}-\vec{u}_{l}\right|\left(\vec{u}_{g}-\vec{u}_{l}\right) .
$$

For the stratified flow regime, it is given by

$$
\vec{F}_{g l}=\frac{1}{2} A_{i} \rho_{m} C_{i}(\phi)\left|\vec{u}_{g}-\vec{u}_{l}\right|\left(\vec{u}_{g}-\vec{u}_{l}\right),
$$

where $C_{D}$ and $C_{i}(\phi)$ are drag force coefficients with

$$
\cos \phi=\frac{\left(\vec{u}_{g}-\vec{u}_{l}\right) \cdot \nabla \alpha}{\left|\left(\vec{u}_{g}-\vec{u}_{l}\right)\right||\nabla \alpha|} .
$$

Ishii [22] and Tentner [18] correlations are used for the calculation of the drag force coefficients. The lift force is given by

$$
\vec{F}_{\text {lift }}=\alpha_{g} \rho_{l} C_{L}\left(\vec{u}_{g}-\vec{u}_{l}\right) \otimes\left(\nabla \otimes \vec{u}_{l}\right),
$$

where $C_{D}$ is the lift force coefficient given by the Tomiyama correlation [23]. The wall lubrication [24] and turbulent dispersion [25] forces are also implemented to consider the lateral motion of gas in liquid. The virtual mass force is expressed as

$$
\vec{F}_{v m}=C_{g}^{v m} \alpha_{g} \alpha_{l} \rho_{m} \frac{\partial\left(\vec{u}_{l}-\vec{u}_{g}\right)}{\partial t},
$$

where

$$
C_{g}^{v m}=\left\{\begin{array}{l}
\frac{\left(1+2 \alpha_{g}\right)}{2\left(1-\alpha_{g}\right)} \text { for } \alpha_{g}<0.5, \\
\frac{\left(3-2 \alpha_{g}\right)}{2 \alpha_{g}} \text { for } 0.5 \leq \alpha_{g} \leq 1
\end{array}\right.
$$

For the interfacial heat transfer coefficients in Eqs. (4) and (5), the following models are used: For bubbly flow [27],

$$
H_{i k}=\frac{k_{k} N u_{b}}{D_{b}} \text { where } N u_{b}=2+0.6 \operatorname{Re}_{b}^{0.5} \operatorname{Pr}_{l}^{0.3} \text {. }
$$

For mist flow [25],

$$
H_{i k}=\frac{k_{k} N u_{d}}{D_{d}} \text { where } N u_{d}=2+0.6 \operatorname{Re}_{d}^{0.5} \operatorname{Pr}_{g}^{0.3} \text {. }
$$

For stratified flow [16],

$$
H_{i k}=S t \rho_{k} c_{p, k}\left|\vec{u}_{g \tau}-\vec{u}_{l \tau}\right|,
$$

where

$$
\begin{aligned}
& S t=0.0045\left(\rho_{g}\left|\vec{u}_{g}\right| \mu_{l} / \rho_{l}\left|\vec{u}_{l}\right| \mu_{g}\right)^{1 / 3}, \\
& \text { and } \vec{u}_{g \tau}-\vec{u}_{l \tau}=\left(\vec{u}_{g}-\vec{u}_{l}\right)-\left[\left(\vec{u}_{g}-\vec{u}_{l}\right) \cdot \frac{\nabla \alpha}{|\nabla \alpha|}\right] \frac{\nabla \alpha}{|\nabla \alpha|} .
\end{aligned}
$$


For the subcooled boiling at the heating surface, a heat partitioning model has been implemented where the heat transfer mechanisms are described as the heat transfer due to surface quenching, evaporation, and fluid convection.

The porous media model is usually applied for a component-scale analysis. Complex configurations of internal structures are simplified by a porosity and permeability for each cell volume and face. When heating surfaces are present in the porous media, the heat conductor is assumed to be uniformly distributed in a cell with the given porosity.

\subsection{Numerical Methods}

In the CUPID code, the finite volume method (FVM) with an unstructured grid is used for complex geometry. A semi-implicit method is applied, where all the terms except the spatial acceleration terms in the momentum equations are solved implicitly and the pressure is calculated in an implicit manner using the conservation equations coupled with scalar equations. This is very useful for a twophase transient calculation where the numerical solution hardly converges with the fully implicit method.

\subsubsection{Discretization of the Governing Equations}

All the independent variables such as pressure, velocity, phasic volume fraction, and internal energy are defined at the cell center. The cell-faced values appearing in the discretized equations are interpolated from the cell-centered values. Note that the cell-faced values are required when the volume integral of a convection term is converted into a surface integral using the Green-Gauss theorem:

$$
\begin{aligned}
& \int_{V} \nabla \cdot(\left(\alpha_{k} \rho_{k} \phi_{k} \vec{u}_{k}\right) d V \\
& \quad=\int_{S} \alpha_{k} \rho_{k} \phi_{k} \vec{u}_{k} \cdot d \vec{S} \approx \sum_{f}\left(\phi_{k} \alpha_{k} \rho_{k}\right)_{f} \vec{u}_{k, f} \cdot \vec{S}_{f}
\end{aligned}
$$

An upwind scheme is used for the interpolation of the cell face convection property, $\left(\phi_{k} \alpha_{k} \rho_{k}\right)_{f}$, and the Rhie-Chow interpolation scheme is applied for the cell face velocity, $\vec{u}_{k, f}$. Cell-faced pressure is used to calculate the pressure gradient at the cell center.

$$
\int_{V} \nabla P d V=\int_{S} P \overrightarrow{i_{i}} \cdot d \vec{S} \approx \sum_{f} P_{f} \vec{S}_{f},
$$

where $P_{f}$ is the pressure obtained from a linear interpolation between the two neighboring cell-centered pressures. The cell-faced gradient appears in the pressure correction equation and the diffusion terms. When the calculation grid is orthogonal, it is approximated as:

$$
\begin{aligned}
\int_{V} \nabla \cdot \gamma \nabla \phi d V & =\int_{S} \gamma \nabla \phi \cdot d \vec{S} \\
& \approx \gamma_{f} \sum_{f} \nabla \phi_{f} \cdot \vec{S}_{f}=\gamma_{f} \sum_{f}\left[\frac{\phi_{j}-\phi_{i}}{\left|d \vec{r}_{i j}\right|}\right] A_{f},
\end{aligned}
$$

where $\left|d \vec{r}_{i j}\right|$ is the distance between the neighboring cells $i$ and $j$ of the face $f$.

\subsubsection{Solution Methods}

The governing equations are solved using a semi-implicit method. For numerical convenience, the left hand side of Eq. (3) is expressed as:

$$
\begin{aligned}
& \frac{\partial}{\partial t}\left(\alpha_{k} \rho_{k} \vec{u}_{k}\right)+\nabla \cdot\left(\alpha_{k} \rho_{k} \vec{u}_{k} \vec{u}_{k}\right) \\
& \quad=\alpha_{k} \rho_{k} \frac{\partial \vec{u}_{k}}{\partial t}+\nabla \cdot\left(\alpha_{k} \rho_{k} \vec{u}_{k} \vec{u}_{k}\right)-\vec{u}_{k} \nabla \cdot\left(\alpha_{k} \rho_{k} \vec{u}_{k}\right)+\vec{u}_{k} \Omega_{k} .
\end{aligned}
$$

The velocity obtained using the explicit method is:

$$
\vec{u}_{k, i}^{*}=\vec{\gamma}_{k, i}^{n}+\beta_{k, i} \nabla P_{i}^{n}
$$

where $\vec{\gamma}_{k, i}^{n}$ includes the explicit convection, diffusion, and body force contributions and $\beta_{k, i}$ is the coefficient of the pressure gradient. The new time velocity is found by:

$$
\vec{u}_{k, i}^{n+1}=\vec{u}_{k, i}^{*}+\beta_{k, i} \nabla \delta P_{i},
$$

where $\delta P_{i}=P_{i}^{n+1}-P_{i}^{n}$. The new time pressure $P_{i}^{n+1}$ is determined to satisfy the continuity and energy equations. Applying the divergence operator $(\nabla \cdot)$ to Eq. (29) and integrating the equation, we obtain:

$$
\sum_{f} \Psi_{k, f}^{n+1}=\sum_{f}\left[\Psi_{k, f}^{*}+\beta_{k, f} \frac{A_{f}}{\left|d \vec{r}_{i j}\right|}\left(\delta P_{j}-\delta P_{i}\right)\right],
$$

where $\Psi_{k, f}$ is the $k$-phase volume flow rate at face $f$ defined as $\Psi_{k, f}=\vec{u}_{k, f} \cdot \vec{S}_{f}$. The temporal volume flow rate, $\Psi_{k, f}^{*}$, is interpolated using the temporal velocities of two neighboring cells, i.e. $\vec{u}_{k, i}$ and $\vec{u}_{k, j}$. Next, the continuity and energy equations are integrated and linearized with respect to the six independent scalar variables of $\left(\delta e_{v}, \delta e_{l}, \delta \alpha_{v}, \delta \alpha_{d}, \delta X_{n c}\right.$, $\delta P)$ to give:

$$
\mathbf{A} \mathbf{x}=\mathbf{s}+\sum_{f} \sum_{k} \mathbf{c}_{k, f} \Psi_{k, f}^{n+1},
$$

where $\mathbf{A}$ is a $6 \mathbf{x} 6$ coefficient matrix, $\mathbf{x}=\operatorname{col}\left(\delta e_{v}, \delta e_{l}, \delta \alpha_{v}\right.$, $\left.\delta \alpha_{d}, \delta X_{n c}, \delta P\right), \mathbf{s}$ is a source vector, and $\mathbf{c}$ is a coefficient vector.

Substituting Eq. (30) into the sixth row of Eq. (31) to eliminate $\Psi_{k, f}^{n+1}$ we obtain the pressure equation as follows:

$$
\delta P_{i}+\sum_{f} C_{f}\left(\delta P_{i}-\delta P_{j}\right)=B_{i}
$$

where

$$
\begin{aligned}
& C_{f}=\frac{A_{f}}{\left|d \vec{r}_{i j}\right|} \sum_{k}\left(\mathbf{A}_{i}^{-1} \mathbf{c}_{k, f}\right)_{6} \beta_{k, f}, \\
& \text { and } B_{i}=\left(\mathbf{A}_{i}^{-1} \mathbf{s}\right)_{6}+\sum_{k}\left(\mathbf{A}_{i}^{-1} \mathbf{c}_{k, f}\right)_{6} \Psi_{k, f}^{*} .
\end{aligned}
$$

This is an $\mathrm{N} \mathrm{x} \mathrm{N} \mathrm{system} \mathrm{of} \mathrm{linear} \mathrm{equation} \mathrm{for} \mathrm{the} \mathrm{new}$ time pressure for $\mathrm{N}$ computing cells, resulting in a nonsymmetric matrix. An iterative method is used to get a solution from the linear equation. In this study, the nonsymmetric matrix solver BiCGSTAB (Bi-Conjugate Gradient STABlized) was used. After solving Eq. (32), the new time phasic velocity $\vec{u}_{k, i}^{n+1}$ and volume flow rate $\Psi_{k, f}^{n+1}$ are determined from Eqs. (29) and (30). 


\subsubsection{Non-orthogonal Grid}

For an orthogonal grid, the cell-faced gradients in the diffusion and pressure equations are simply approximated as shown in Eq. (26). This is because the line connecting cell centers $i$ and $j$ passes through the cell face center as shown in Fig. 2. When the grid is non-orthogonal or irregular, the line connecting cell centers may not pass through the cell face center. In this case, the cell-faced gradient is defined between the new coordinates $i^{\prime}$ and $j^{\prime}$ where the values are interpolated using the gradient at the cell center.

$$
\phi_{i^{\prime}}=\phi_{i}+\nabla \phi_{i} \cdot\left(\vec{x}_{i^{\prime}}-\vec{x}_{i}\right)
$$

Using Eq. (33) the cell-faced gradient in Eq. (26) becomes:

$$
\begin{aligned}
& \gamma_{f} \sum_{f} \nabla \phi_{f} \cdot \vec{S}_{f}=\gamma_{f} \sum_{f}\left[\frac{\phi_{j^{\prime}}-\phi_{i^{\prime}}}{\left|d \vec{r}_{i j}\right|}\right] A_{f} \\
& \quad=\gamma_{f} \sum_{f}\left[\frac{\phi_{j^{\prime}}-\phi_{i^{\prime}}}{\left|d \vec{r}_{i j}\right|}+\frac{\nabla \phi_{j} \cdot\left(\vec{x}_{j^{\prime}}-\vec{x}_{j}\right)-\nabla \phi_{i} \cdot\left(\vec{x}_{i^{\prime}}-\vec{x}_{i}\right)}{\left|d \vec{r}_{i j}\right|}\right] A_{f}
\end{aligned}
$$

Since the diffusion terms are calculated explicitly, Eq. (34) is easily implemented. However since the pressure correction equation, i.e. Eq. (32), is calculated implicitly, the grid non-orthogonality is taken into account in the equation iteratively. In the first step, the pressure correction equation is solved for $\delta P_{i}$ where the non-orthogonal terms in Eq. (34) are neglected. In the second step, a second pressure correction, $\delta \delta P_{i}$, is made to account for the error in the first step.

$$
\begin{aligned}
\delta \delta P_{i}+\sum_{f} C_{f} & \left(\delta \delta P_{i}-\delta \delta P_{j}\right) \\
& =-\sum_{f} C_{f}\left[\delta P_{i} \cdot\left(\vec{x}_{i^{\prime}}-\vec{x}_{i}\right)-\delta P_{i} \cdot\left(\vec{x}_{j^{\prime}}-\vec{x}_{j}\right)\right]
\end{aligned}
$$

The right-hand side of Eq. (36), given from the first step, becomes zero when the grid is orthogonal. In this case, the second pressure correction is not necessary.

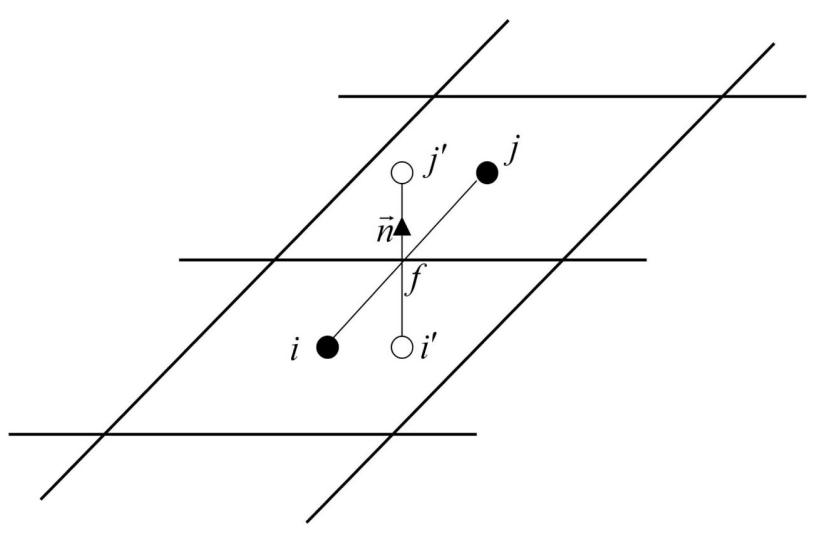

Fig. 2. Non-orthogonal Grid

\section{VALIDATION OF THE CUPID}

Numerical stability and accuracy of the CUPID code has been verified using conceptual problems [9]. Each of the problems was designed so that a specific term in the governing equations or a specific algorithm could be verified. The validation is the assessment of the physical accuracy of a computational model based on comparisons between computational simulations and experimental data. The open media model of the CUPID code, which is used for a CFD scale analysis, was validated through an assessment of a downcomer boiling experiment. Then the porous media model used for a component-scale analysis was validated using an experiment on the moderator flow in a Calandria vessel.

\subsection{Analysis of Downcomer Boiling}

The DOBO test facility was designed to simulate the downcomer boiling phenomena that may occur in the lower downcomer region in a PWR during the reflood phase of a postulated LBLOCA (Large Break Loss Of Coolant Accident) [10]. The DOBO facility consists of a test section, a condenser, a heat exchanger, a drain pump, a mixing tank, a pre-heater and an injection pump, as shown in Fig. 3(a). The test section has rectangular duct geometry whose dimensions are $6.4 \mathrm{~m}$ high, $0.25 \mathrm{~m}$ wide and $0.30 \mathrm{~m}$ deep. The height and width of the test section are the same as those of the APR1400 downcomer, but the circumference is reduced by 47.08 -fold. One of the four side walls of the test section incorporates 207 cartridge heaters inside it to simulate the stored energy release from the reactor vessel wall. The heated region starts $0.3 \mathrm{~m}$ above the inlet, and ends $1.0 \mathrm{~m}$ below the outlet. Fig. 3(b) presents the schematic diagram of the test section. The DOBO test was performed with four different heat fluxes; the DOBOR2-1 test conducted with $70.8 \mathrm{~kW} / \mathrm{m} 2$ was selected for the current simulation because it is the closest value to the predicted heat flux at the reflood phase of a LOCA.

Fig. 4 shows the boundary conditions and the computation mesh. The test section was modeled in two dimensions with the symmetric boundary condition. The experimental results averaged along the depth direction justified this simplification because it showed the overall flow behavior, such as the void fraction profile and its transition, as well as the circulation of the liquid flow, were well preserved in the averaged quantities. A total of $3096(24 \times$ 122 for the duct) cells were used for the present calculation.

The calculated void fraction distributions shown in Fig. 5(a) are compared with the experimental data in Fig. 5 (b) at three different elevations $(1.53 \mathrm{~m}, 3.53 \mathrm{~m}$ and 4.53 $\mathrm{m})$. At an elevation of $1.53 \mathrm{~m}$ (Elevation-1), the wall peaking of the void fraction profiles was obtained at the experiment and the simulation. At an elevation of $3.53 \mathrm{~m}$ (Elevation-2), a significant difference in the void fraction profile was found between the experiment and the calculation. In the experiment, more bubbles were found in the center 


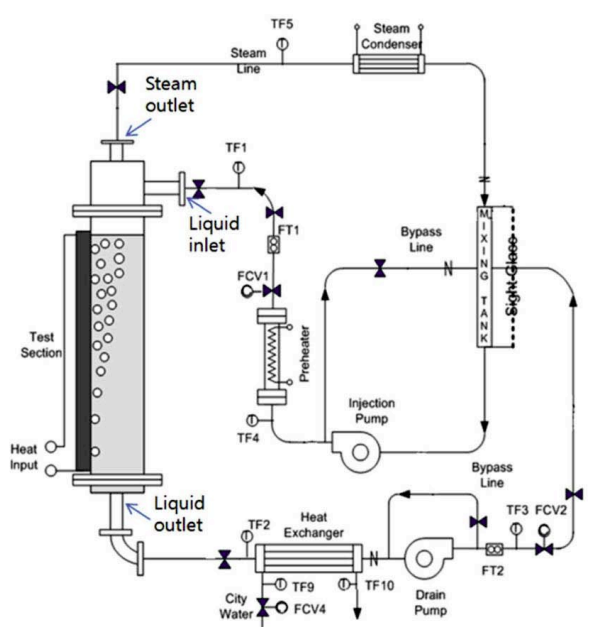

(a) The test loop

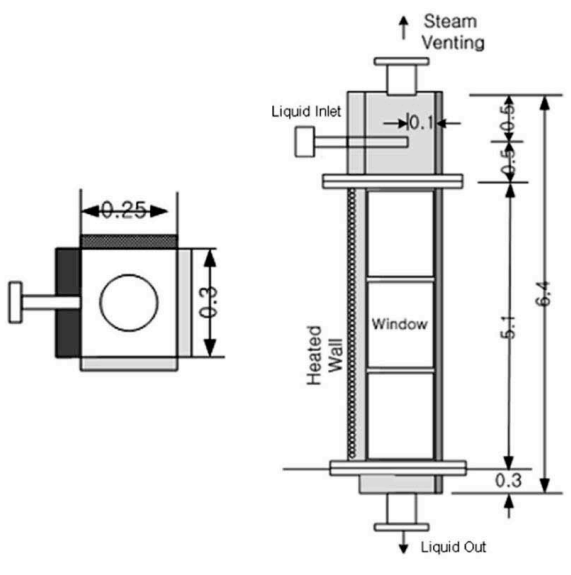

(b) The test section

Fig. 3. The Schematic of the DOBO Test Facility
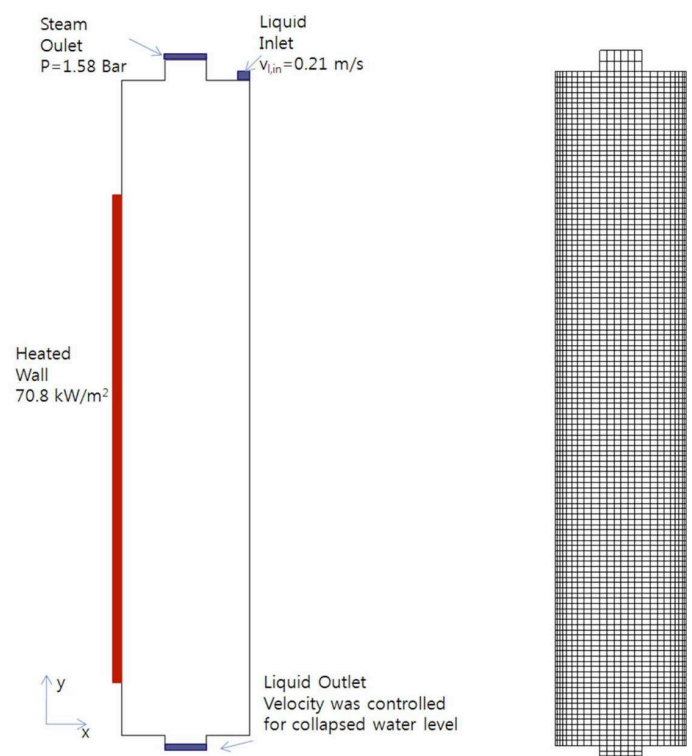

Fig. 4. Boundary Conditions of the Calculation and the Computation Mesh for DOBO

region of the channel, and the core peaking profile of the void fraction was observed. In the calculation, however, CUPID predicted a similar profile with that of Eleveation1 where the transition from the wall peaking to the core peaking had not yet started. This is due to the fact that the calculated bubble size was not large enough to change the direction of the lift force; the bubbles kept residing on the heated wall. The core peaking of the void fraction profile was reasonably well reproduced by the CUPID code at an elevation of $4.53 \mathrm{~m}$ (Elevation-3). As shown in Fig. 5(a), the transition from the wall peaking to the core peaking started at approximately $4.2 \mathrm{~m}$ and afterwards, the location of the void fraction peak moved to the center of the channel. The predicted bubble diameter increased with void fraction along the elevation and became larger than the criterion for the lift force direction.

In the downcomer boiling analysis, averaged properties, such as the averaged void fraction and pressure, play an important role for the reactor core cooling since they are related to the hydraulic head of the accumulated water, i.e. the driving force of water reflooding. For this reason, the averaged properties were compared between the calculation and the experiment in Figs. 6 and 7. As shown in the figures, CUPID properly predicted the averaged void fraction and pressure. The maximum difference between the predicted and measured pressure was less than $2 \%$.

\subsection{Moderator Flow Analysis in a Calandria Vessel}

In this section, the CUPID code was validated using the single-phase flow experiments that were performed at Stern Laboratories Inc.[11] to study the moderator flow in a Calandria vessel. In this experiment, the heat-generating tube bundle was located in the core of the thin "slice-typed" cylinder to represent the Calandria vessel. The tube bundle consisted of 440 inconel heaters with a diameter of $33 \mathrm{~mm}$ and a $71.5 \mathrm{~mm}$ pitch among the tubes. The total power into the bundle was $100 \mathrm{~kW}$. A pair of coolant inlet nozzles were installed, each of which was a thin planar jet with 6 $\mathrm{mm}$ width and located $50 \mathrm{~mm}$ away from the inner wall (see Fig. 8). Though the nozzles were originally designed to adjust their width from $6 \mathrm{~mm}$ to $18 \mathrm{~mm}$ and their angle up to $15^{\circ}$ from the vertical direction toward the center, they were fixed at $6 \mathrm{~mm}$ width in the vertical direction for the experiment. The outlet nozzle was $15 \mathrm{~mm}$ wide at the bottom of the test section. Fig. 8 shows a schematic of the test section and corresponding computational mesh for the simulation. Among various tests, the nominal-condition case [11], known to represent the normal operation in a real CANDU reactor, was selected in this assessment. In the test, the flow rate was $2.4 \mathrm{~kg} / \mathrm{s}$ with a total power of 


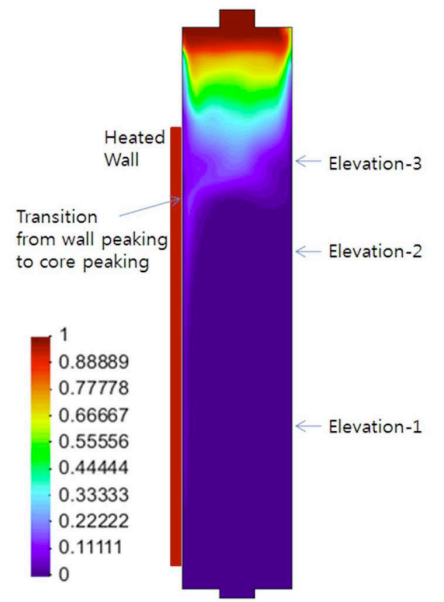

(a) Void fraction distribution

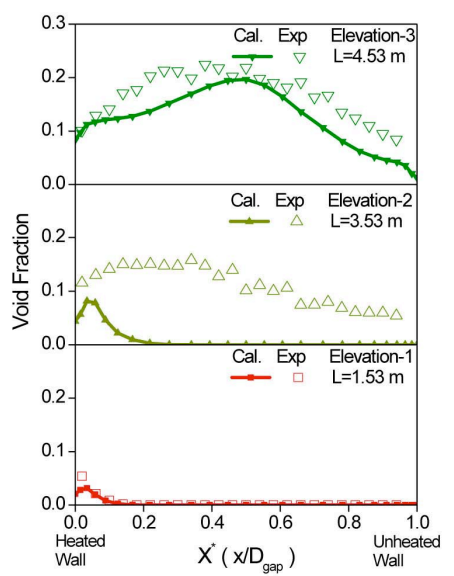

(b) Comparison with the experimental data

Fig. 5. Calculation Result for DOBO

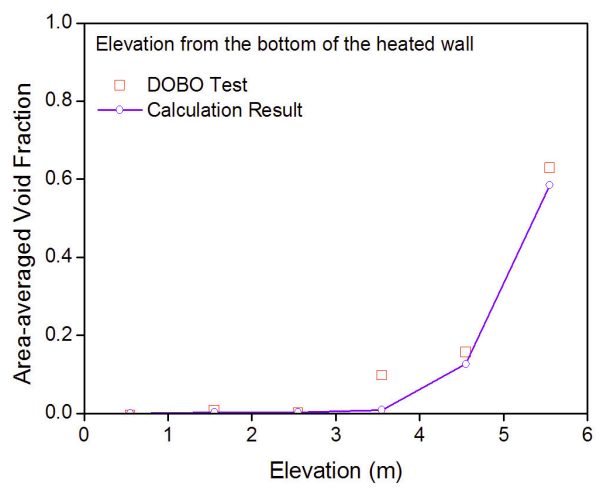

Fig. 6. Area Averaged Void Fraction along the Elevation

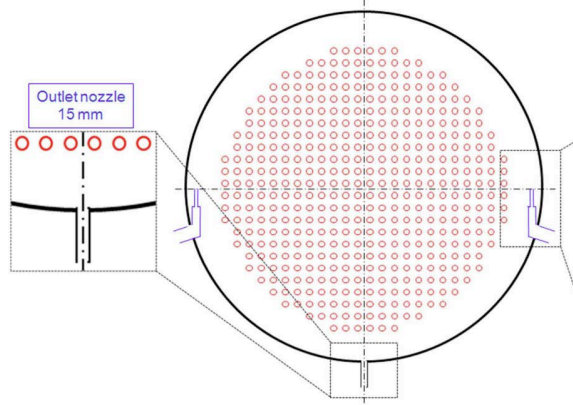

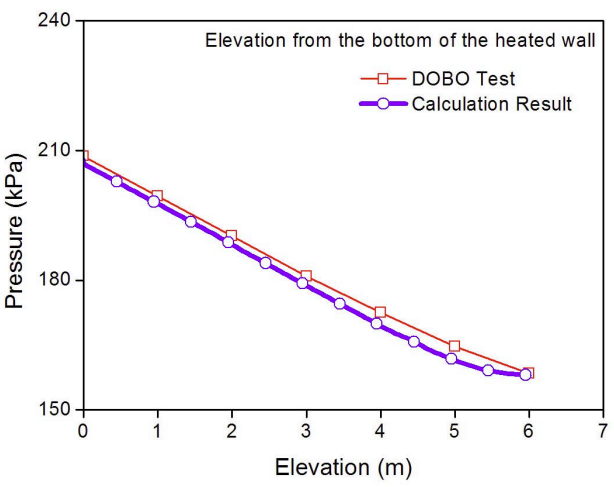

Fig. 7. Pressure along the Elevation (a) Schematics

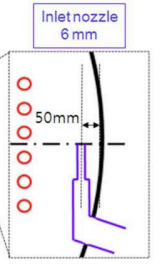

Fig. 8. Schematics and Grid System for the STERN Moderator Experiment.
$100 \mathrm{~kW}$. The inlet water temperature was constant at $55^{\circ} \mathrm{C}$.

To avoid the complexity of generating computational geometry around the Calandria tube bundles, a porous media approach was applied for that region and the flow resistance inside the porous media zone was modeled using an empirical correlation [28]. A combination of polyhedral mesh and bent structured mesh system was applied in this simulation as shown in Fig. 8(b); the bent structured mesh was used for the outer fluid region and the polyhedral mesh for the inner porous region. The polyhedral mesh system has the advantage of keeping orthogonality among neighboring cells. In this study, 7800 meshes were applied to perform the validation against the experimental results. The inlet nozzle was assigned with a single mesh layer to minimize uncertainty in a momentum flux.

In this calculation, the heat is not supplied during the 


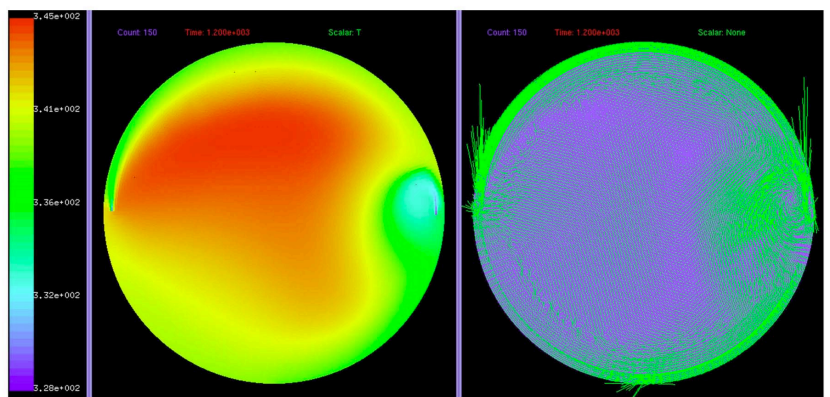

Fig. 9. Contours of the Liquid Temperature and Velocity Vectors for the Nominal Case.

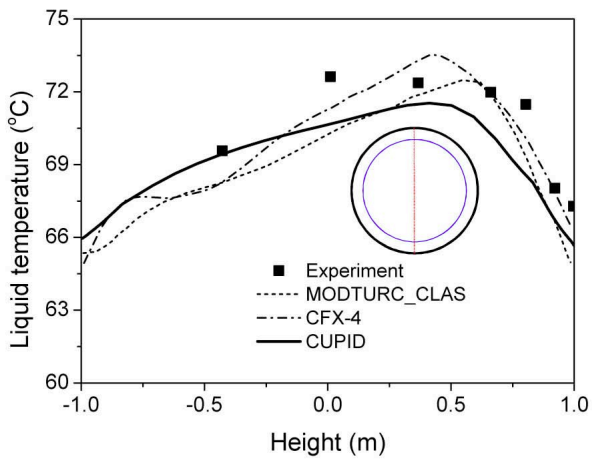

(a) Vertical profile at $\mathrm{x}=0$

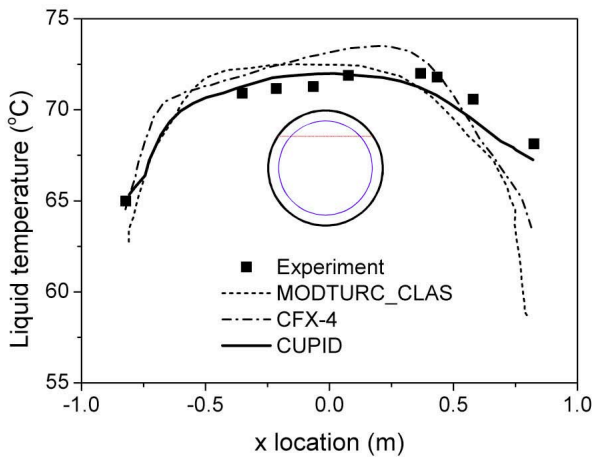

(b) Horizontal profile at $\mathrm{y}=0.57$

Fig. 10. Profile of the Liquid Temperature for the Nominal Case

first 50 seconds. Thereafter, it is linearly loaded for 100 seconds and then maintains the rated value. Fig. 9 shows the contour of the liquid temperature and velocity vectors for the nominal case. Since there is no heat generation at the beginning of the simulation, the flow pattern showed exact $y$-axis symmetry. As the heat is supplied into the porous zone, the buoyant force is gradually generated. Consequently, the y-axis symmetry is collapsed at an arbitrary state due to the instability between the advection from the coolant injection against the buoyant force. The stagnation point at the top is tilted at either direction, which can be affected by any small numerical perturbation that comes from the flow instability. Once the flow symmetry has collapsed, an enlarged impinging jet continuously developed up to near the opposite nozzle. Finally, the asymmetric flow pattern maintained the balance between flow momentum and buoyancy-driven force. This steady state is called the mixed flow regime of forced and natural convection [28, 29].

The liquid temperature distributions for both vertical and horizontal direction are plotted in Fig. 10. These are compared with those from other calculations and experimental data. From the vertical profile in Fig. 10(a), one notices that the result of CUPID is in good agreement with the others. However, at the mid zone $(y \approx 0.0)$ and upper fluid layer $(y \geq 0.85)$, the CUPID code under-predicts the liquid temperature. A local maximum liquid temperature occurs at the upper porous zone $(0.3 \leq y \leq 0.6)$. The hori- zontal distribution of the liquid temperature at $y=0.57$, obtained using CUPID, fits the experiment quite well as shown in Fig. 10(b), while the other numerical results show relatively low liquid temperature in the right-hand region. As shown in Fig. 9, the dominant liquid jet from the lefthand nozzle causes the stagnation point to be close to the right-side nozzle. This suppresses the opposite jet from becoming a circular vortex. Since the heat is generated beneath the enlarged jet layer, the enlarged vortical structure of the flow injection makes the liquid temperature in the right-had side at $y=0.57$ higher than that from the other calculation.

\section{MULTI-SCALE THERMAL HYDRAULIC ANALYSIS}

The multi-scale analysis method can be either an "explicit (weak)-coupled" or an "implicit (strong)-coupled" method. In an explicitly-coupled method, the simulation results obtained from a larger length-scale mesh are used as boundary conditions for a refined calculation with a smaller length-scale mesh. On the other hand, in an implicitly-coupled method, a smaller length-scale code is coupled with a larger length-scale code at the source level. In this work, a CFD-scale code, CUPID, has been coupled with a system-scale code, MARS, implicitly by solving the pressure equations of the two codes simultaneously. 

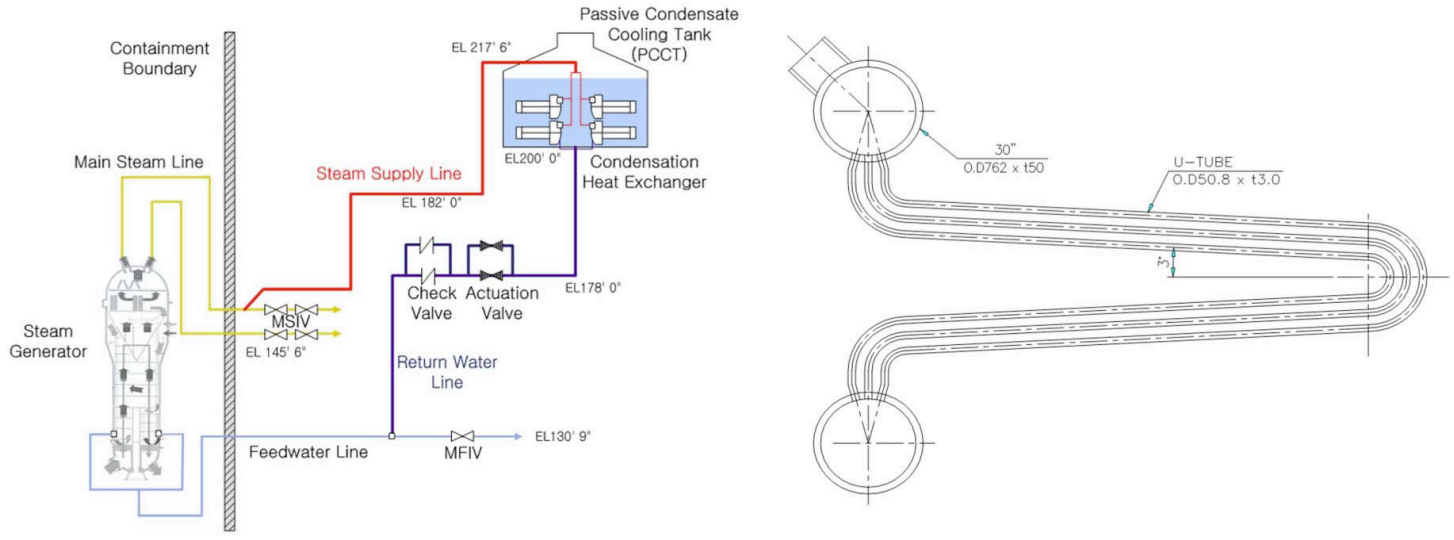

Fig. 11. Schematic Diagram of the APR+ PAFS and the PCHX

\subsection{Explicit Coupling Method}

The CUPID code provides both component and CFDscale analyses by choosing different physical models. Porous and open media models are usually applied for component- or CFD-scale analyses, respectively. In this study, the single- and two-phase natural circulations in PCCT of the PASCAL test are simulated in a component scale and the local boiling heat transfer outside the heat exchanger tube is calculated in a CFD scale. The simulation results of the PCCT are used as boundary conditions for the local calculation of the heat exchanger tube.

\subsubsection{Component-scale analysis: Assessment of the PASCAL PCCT Test}

The PAFS is the auxiliary feedwater system which is capable of condensing steam generated in a steam generator and re-feeding the condensed water to the stream generator by gravity as illustrated in Fig. 11 [30]. It incorporates 240 horizontal U-tubes (PCHX) submerged in a large water pool (PCCT). The steam flows through the tubes and condenses afterwards due to the heat transfer from the PCHX to the PCCT. The water level decreases as it boils. The steam generator feeding can be continued until the water level is decreased lower than the PCHX elevation. The system was designed to provide cooling capability for the whole decay heat at least 8 hours in the event of an accident.

To investigate the cooling and operational performance of the PAFS, the condensation heat transfer in the PCHX, and natural convection in the PCCT, the PASCAL test facility was constructed [14]. The steam generator supplies saturated steam at 7.4 MPa. The PCHX and the PCCT of the PASCAL facility were designed based on a volumetric scaling methodology (Nahavandi, 1979). It simulates a single tube among 240 tubes in the prototype, that is, the volumetric scaling ratio of the facility is $1 / 240$. The volume of the PCCT was reduced to $1 / 240$ of the prototype as well. In order to preserve the natural convection flow in the PCCT, the height of the pool is determined to be the

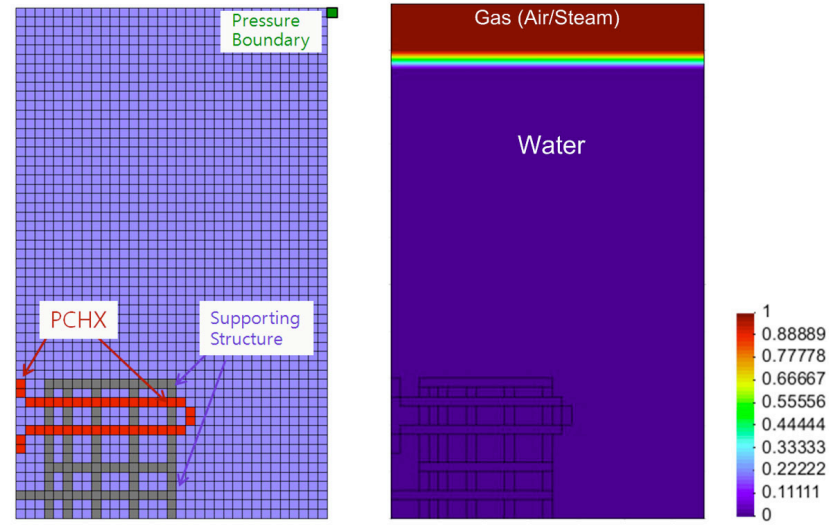

Fig. 12. Computational Domain and Initial Condition of Void Fraction

same as that of the prototype. The width of the PCCT in the PASCAL facility is $6.7 \mathrm{~m}$, which is half of that of the prototype. The depth of the PCCT is $0.112 \mathrm{~m}$, which is equivalent to $1 / 120$ of the prototype.

In order to investigate the natural circulation and the boil-off phenomena in the PCCT of the PASCAL test facility, a transient calculation was performed using the CUPID code. The problem time was 30,000 seconds; the same as with the experiment. It was modeled in two dimensions using the porous media model and simulated using a total of $1815(33 \times 55)$ meshes. Fig. 12 shows the computational domain of the calculation and the initial condition of the void fraction. The initial PCCT water level was $9.8 \mathrm{~m}$ and the region above the free surface was filled with air. The initial liquid and gas temperatures were both $40{ }^{\circ} \mathrm{C}$. At the top right cell of the PCCT, a pressure boundary condition was imposed as indicated in Fig. 12. It was maintained at $0.1013 \mathrm{MPa}$ during the transient. The heat released from the PCHX was simulated by imposing a volumetric heat source from the measured data. The important phenomena observed in the experiment are illustrated in Fig. 13.

The water level was elevated from $9.8 \mathrm{~m}$ to $10.4 \mathrm{~m}$ owing to thermal expansion. For the initial 7000 seconds, 


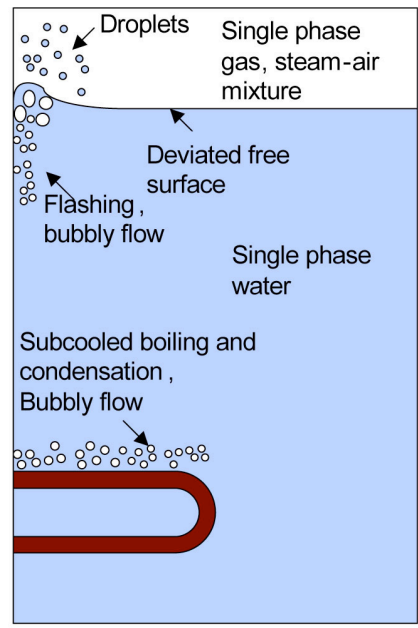

Fig. 13. Major T/H Phenomena in the PCCT

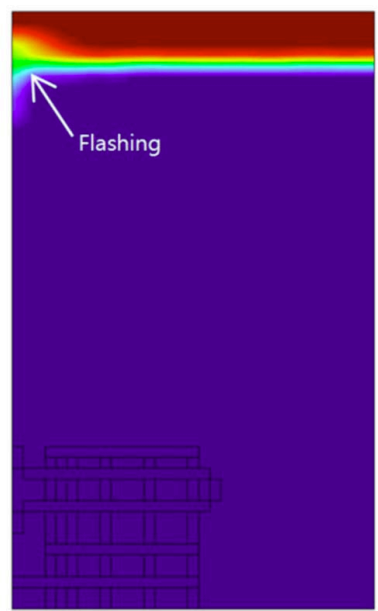

Fig. 14. Calculation Results: Void Fraction (7500s)

the single phase natural circulation was continued because the liquid subcooling had been maintained, but after that, a two-phase region appeared near the free surface due to flashing as presented in Fig. 14. Since the pressure near the free surface was close to the atmospheric one, the liquid became superheated as it flowed upward and then, a flashing was initiated. The start of the two-phase natural circulation greatly enhanced the liquid velocity. The two-phase mixture level swells due to the flashing as shown in Fig. 14, which agrees with the experimental observation.

The void fraction distributions after 7000 seconds are plotted in Fig. 15 at every 11000 seconds. The result showed that the heat removal by the boil-off can last longer than 8 hours (28800 seconds) as it was designed. In Fig. 16, the liquid temperature transients at two different positions were compared between the experiment and the calculation; one is located $0.3 \mathrm{~m}$ below the initial free surface elevation and the other $0.1 \mathrm{~m}$ below the PCHX. The liquid temperature gradually increased from $40{ }^{\circ} \mathrm{C}$

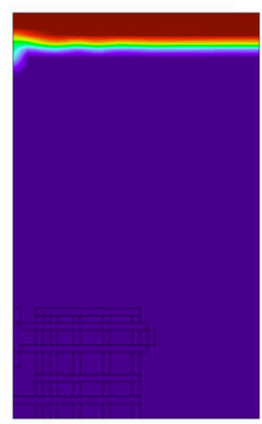

(a) $7000 \mathrm{sec}$

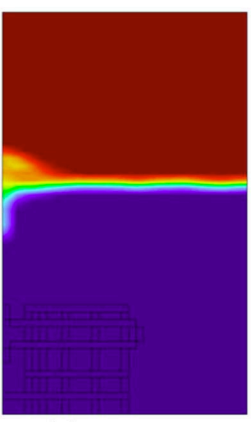

(c) $18000 \mathrm{sec}$.

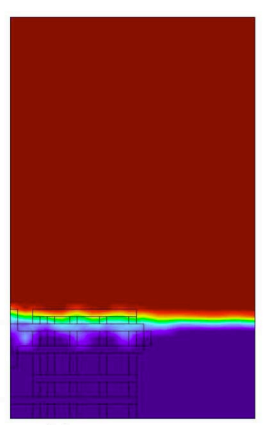

(e) $29000 \mathrm{sec}$.
Fig. 15. Water Level Decreases Due to the Boil-off

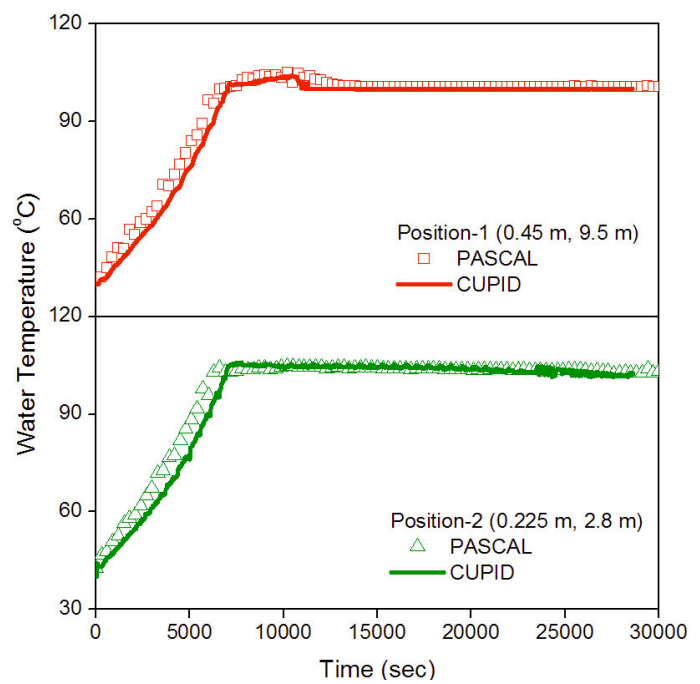

Fig. 16. Liquid Temperatures Transient in the Experiment and the Calculation

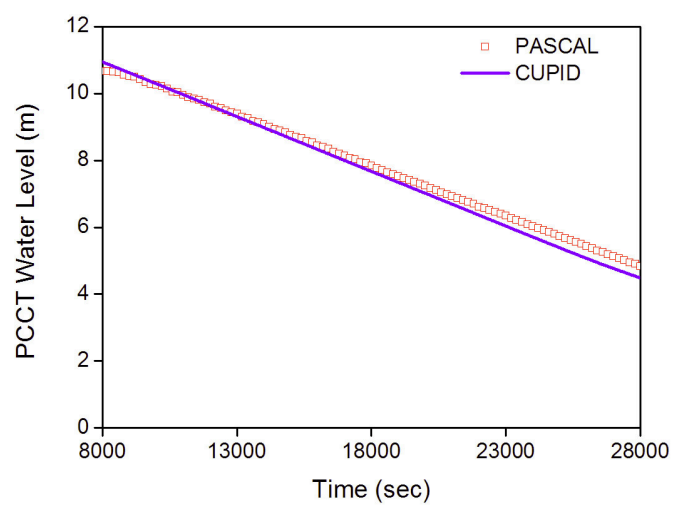

Fig. 17. Water Level Decreasing Rate

with the heat release from the PCHX and then made a plateau as it reached the saturation temperature.

Fig. 17 presents the water level decreasing rate due to the boil-off. The decreasing rate error is $6.4 \%$ for the whole transient. Despite the fact that the boiling-off had started, there existed a certain portion of the sensible heat transfer. 

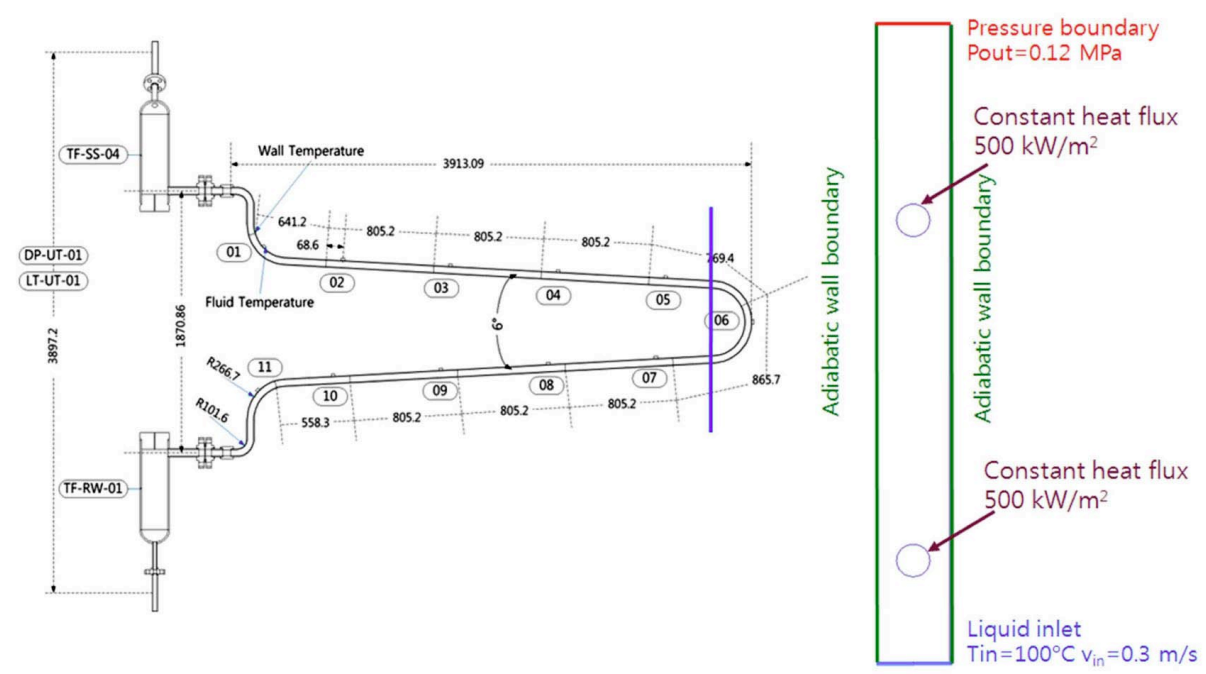

Fig. 18. Computational Domain

This is because the water near the heat exchanger still remained in a subcooled state due to the hydraulic head of the water. Therefore, the under-estimated sensible heat transfer could result in an error in the decreasing rate. The error could also be caused by the estimation of the heat loss during the two-phase natural circulation. Since the experiment provides neither heat loss rate nor the wall temperature measurement data, the heat loss rate was estimated by comparing the liquid temperature increasing rate and the imposed power. However, in the two-phase conditions, the error can be enlarged with the distorted and fluctuating water level. In spite of this error, the overall boiloff procedure was reasonably well reproduced by CUPID.

\subsubsection{CFD-scale Analysis: Boiling Heat Transfer Outside the Tube Bundle of PCCT}

The boiling heat transfer outside the tube bundle of the PCCT was analyzed in a CFD scale using the PCCT simulation results. The liquid temperature, velocity and pressure obtained from the PCCT simulation were imposed for the inlet and outlet boundary conditions of this analysis. Fig. 18 shows the computational domain. A cross-section along the depth direction at a location was modeled in two dimensions, where the bending part of the heat exchanger starts. A total of 6933 polygonal meshes were used for the analysis. Fig. 19 shows the calculation results of the void fraction, the liquid temperature, and liquid velocity distributions. The calculated heat exchanger wall temperature is compared with the experimental result as well.

A similar approach with the above analysis was applied to the unit cell of the heat exchanger tube bundle. Fig. 20 shows the arrangement of the heat exchanger tube bundle. Since they are not uniformly arranged in terms of space, the local volumetric heat source in the tube bundle is larger than that in the PASCAL facility. It is required to verify
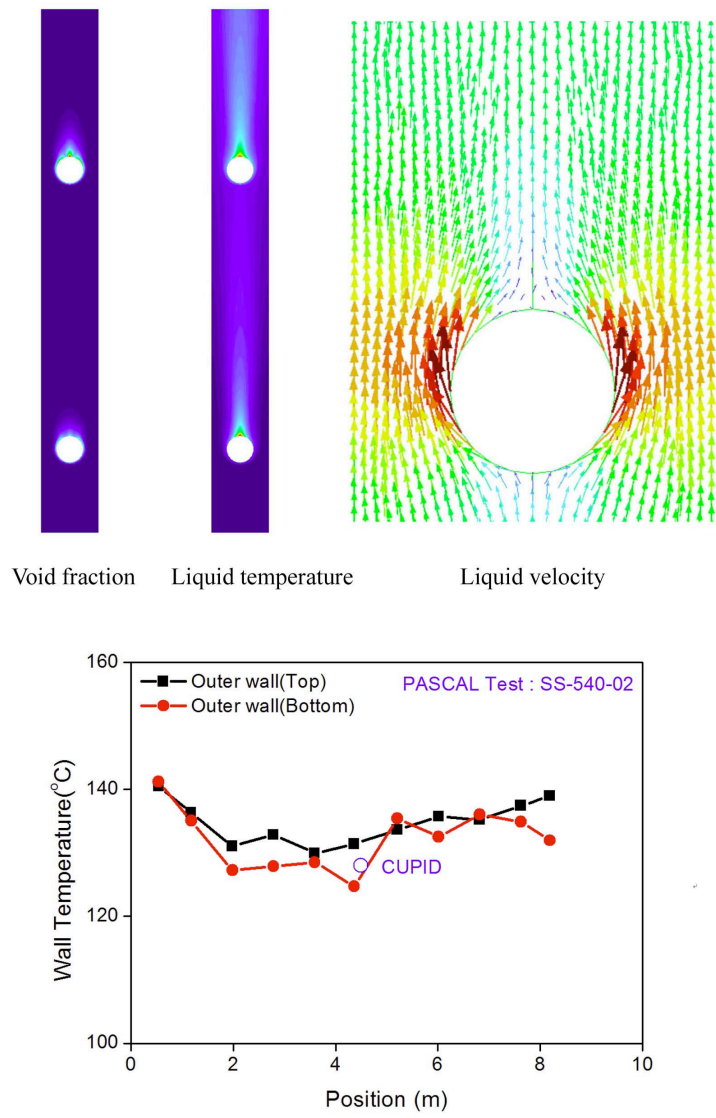

Fig. 19. Calculation Result (Single Tube)

that the distortion of the local power does not cause a significant distortion of the heat exchanger wall temperature in order to ensure that the cooling capability of the heat exchanger evaluated by the PASCAL facility is applicable to the bundle geometry. For this reason, the unit cell of 

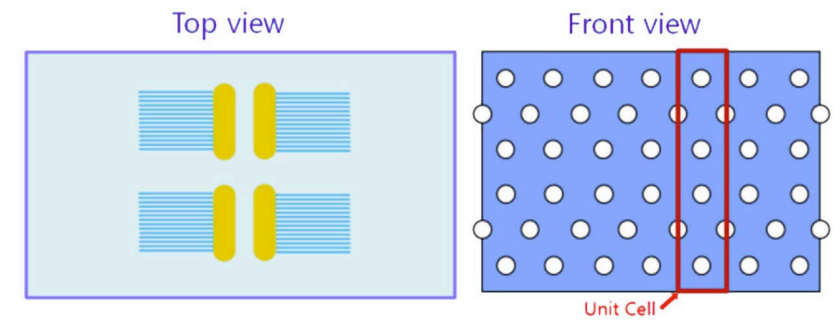

Fig. 20. Arrangement of the Tube Bundle (Simplified)

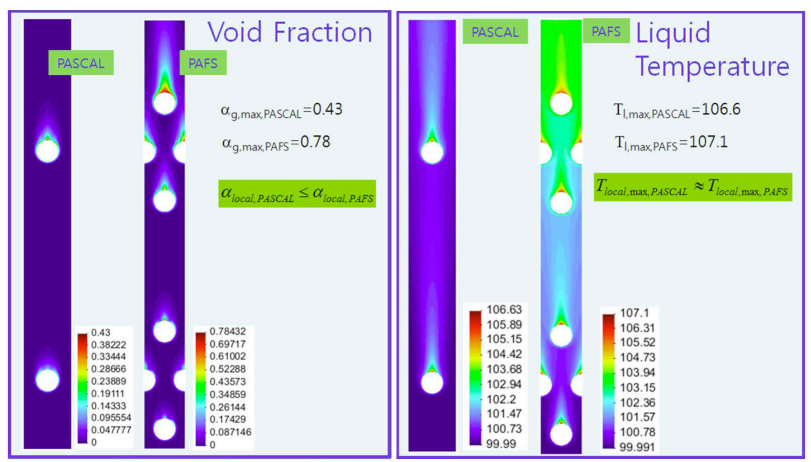

Fig. 21. Calculation Result (Single Tube vs. Unit Cell)

the tube bundle was simulated with a periodic boundary condition on the side boundaries and a total of 15282 polygonal meshes were used. The same flow conditions were imposed on the boundaries and this includes conservativeness because the natural circulation rate would be higher in the bundle case due to the higher local volumetric power. Fig. 21 shows the calculation results of the void fraction and the liquid temperature. Compared to the single tube calculation, a higher void fraction was obtained with the higher volumetric power. The maximum void fractions on the heat exchanger tube were 0.43 and 0.78 at the single tube and bundle calculations, respectively. However, the subcooled boiling flow patterns were maintained in the bundle case, and therefore, the maximum temperatures of the liquid and the heat exchanger surface were comparable with the single tube calculation results. This implies that the cooling capability of the heat exchanger tube bundle by the natural circulation is comparable with that in the PASCAL facility.

\subsection{Implicit Coupling Method}

In an implicitly-coupled method, a smaller scale code is coupled with a larger scale code at the source level. In this paper, a CFD scale code, CUPID, has been coupled with a system scale code, MARS, implicitly by solving the pressure equations of the two codes simultaneously. Using the coupled code, we can simulate a specific part of a thermal-hydraulic system with CUPID and the rest of the system with the system code, which can provide the system code with a local zoom function.

\subsubsection{Coupling Algorithm}

The system code, MARS [3], was chosen for this coupling because its hydrodynamic model adopts a two-fluid model for a two-phase flow and its numerical scheme is similar to the corrector step of the CUPID code. Referring to the previous experiences [31], the MARS-CUPID coupling was performed.

Let us consider a flow system that is divided into a three-dimensional region (modeled by CUPID) and several one-dimensional regions (modeled by MARS) with $N C$ interface junctions (or connections). For convenience, let us define $C i$ and $M i$ as the index numbers of $i$-th interfacing cells in the CUPID and MARS regions, respectively. In the MARS code, cell $C i$ is treated as a "CUPID boundary volume (cupvol)", whose scalar variables are updated every time-step by CUPID. In the CUPID code, cell Mi is regarded as a sink that is implicitly coupled. The pressure correction matrices, which are set up in each module, are coupled via the momentum modeling at the interfaces and solved simultaneously.

The momentum balance at the interface junction from cell $C i$ to $M i$ is modeled by the MARS code, where the old time-step variables of cell $C i$ are transferred from CUPID. Then, the phasic volume flow at the $i$-th interface junction $\Psi_{k, f}^{n+1}$ is given by

$$
\Psi_{k, i}^{n+1}=\alpha_{k, i}+\beta_{k, i}\left(\delta P_{C i}-\delta P_{M i}\right) .
$$

Due to the MARS-CUPID connections, the pressure correction equation of MARS involves additional unknown terms that include the unknown velocities at the interfaces:

$$
\mathbf{A}_{M} \delta P_{M}=\mathbf{b}_{M}+\sum_{i=1}^{N C}\left(\gamma_{l, i} \Psi_{l, i}^{n+1}+\gamma_{g, i} \Psi_{g, i}^{n+1}\right)
$$

where $\gamma_{l}$ and $\gamma_{g}$ are coefficient vectors. Likewise, the pressure correction equation of CUPID is also changed due to the connection:

$$
\mathbf{A}_{C} \delta P_{C}=\mathbf{b}_{C}+\sum_{i=1}^{N C}\left(\boldsymbol{\kappa}_{l, i} \Psi_{l, i}^{n+1}+\boldsymbol{\kappa}_{g, i} \Psi_{g, i}^{n+1}\right),
$$

where $\boldsymbol{\kappa}_{l}$ and $\boldsymbol{\kappa}_{g}$ are coefficient vectors. Sustituting Eq. (37) into Eqs. (38) and (39), a coupled pressure correction equation for the whole system can be established. The coupled equation is solved by using a domain decomposition method. After solving it, the remaining numerical sequences are completed in each code.

\subsubsection{Coupled Simulation of ROCOM Test}

For a detailed investigation of the thermal hydraulic behavior inside the reactor pressure vessel (RPV), complementary tests on the coolant mixing were conducted at the ROCOM test facility [15]. In order to validate the coupled code, MARS-CUPID, the ROCOM test 1.1 was simulated.

The ROCOM RPV was modeled with 37,068 3-dimensional hexahedral meshes using the CUPID code (Fig. 22), and the pipes, pumps, and valves of the four loops were 
modeled with 40 one-dimensional volumes using the MARS code (Fig. 23). The internal structures in the RPV were modeled as porous media, where a zero equation

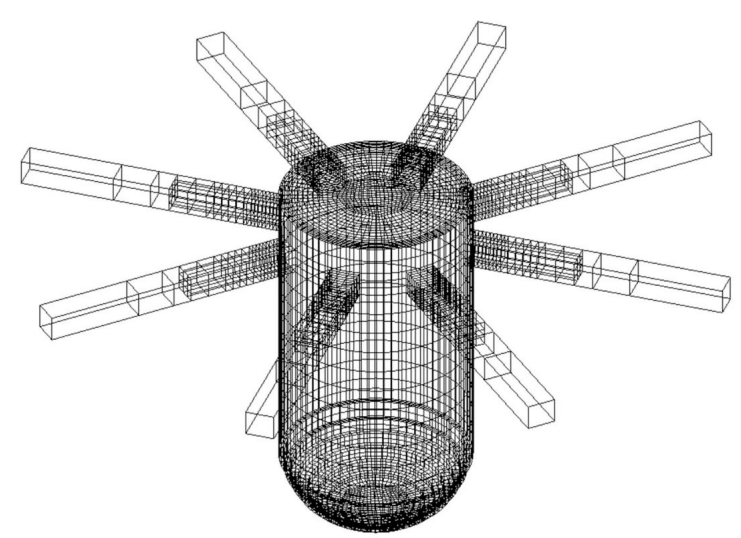

Fig. 22. Hexahedral CUPID Meshes for ROCOM RPV model is adopted for turbulence modeling. At first, a steady state calculation was carried out to simulate the initial state of the test where three out of four reactor coolant pumps are in operation. The system pressure is 40 bars and the coolant temperature is uniform at $500 \mathrm{~K}$. At 100 seconds, the reactor coolant pump which was initially disabled starts to deliver cold water of $400 \mathrm{~K}$ at a flow rate four times that of the other pumps. This cold water injection continues for 90 seconds, and then all the pumps were stopped.

The calculated temperature distribution at 50 seconds after the start of the cold water injection into the RPV is presented in Fig. 24. This figure shows that the cold water injected into one cold leg flows via the downcomer to the core and mixes with hot water during the transient. The calculated coolant temperatures inside and outside the downcomer and at the core inlet are compared to the measured ones in Fig. 25. The downcomer coolant temperatures decrease around 10 seconds after the cold water injection. The core average coolant temperature decrease rate is

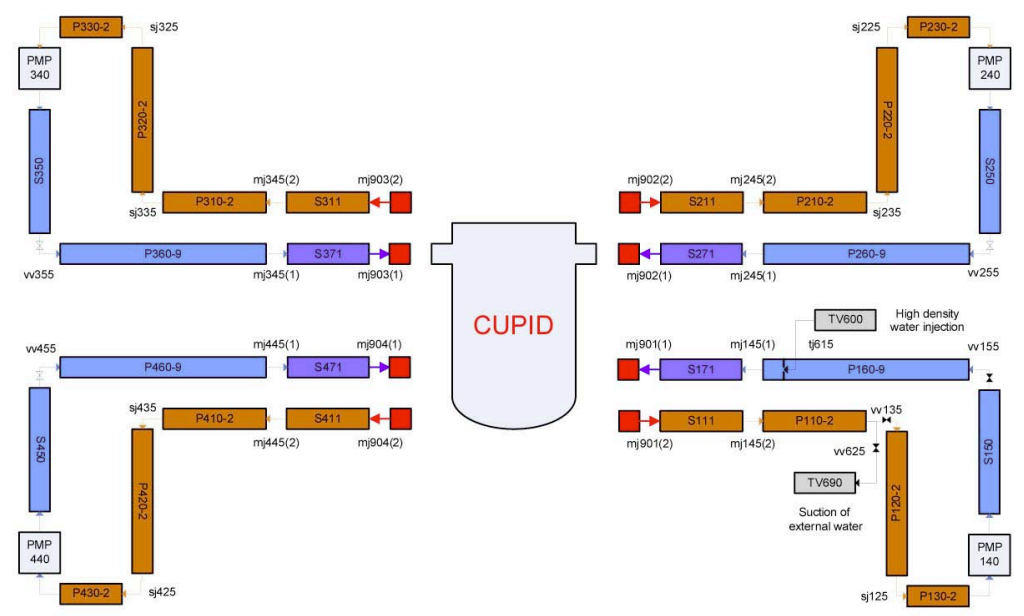

Fig. 23. 1-D MARS Volumes for ROCOM 4 Loops

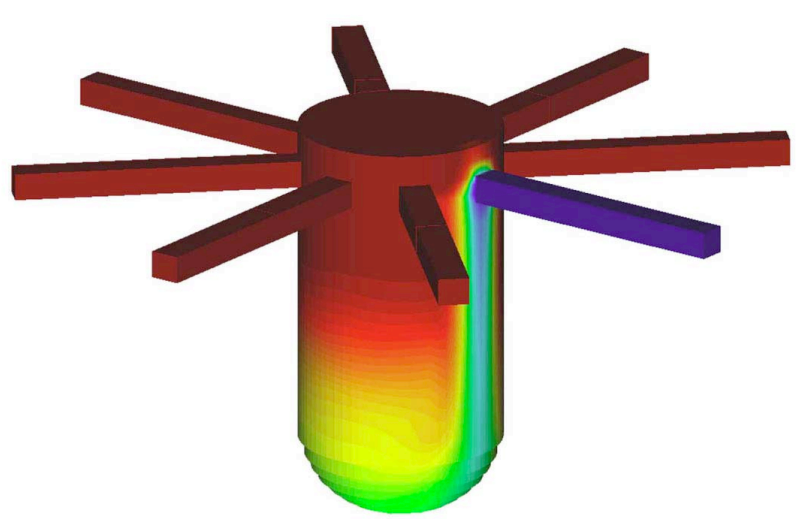

Fig. 24. Coolant Temperature Distribution 50s after the Cold Water Injection

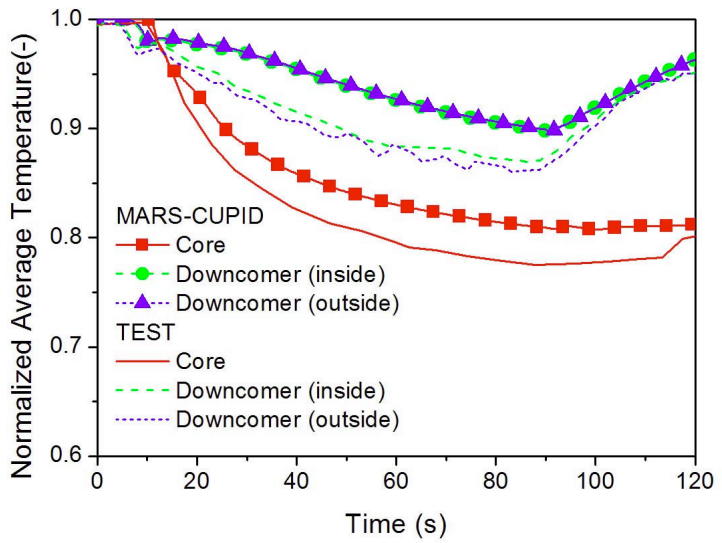

Fig. 25. Comparisons of the Average Temperatures 
larger than that of the downcomer since the coolant is not fully mixed in the core. The coolant temperatures rapidly recover after 90 seconds when the cold water injection stops, but the recovery rate of the core inlet temperature is relatively low. These overall trends of the calculated temperatures agree with those of the measured ones although they are a little higher than the measured ones due to numerical diffusion.

\section{CONCLUSIONS}

The CUPID code had been developed for the analysis of transient two-phase flows in PWRs, where three-dimensional two-fluid, three-field governing equations are adopted and discretized using the finite volume method on unstructured grids. A semi-implicit method is used to obtain the numerical solution. The numerical method of the CUPID code has been verified in previous studies.

In this paper, the CUPID code has been validated against experiments related to nuclear reactor thermalhydraulic issues, such as downcomer boiling and the mixed moderator flow pattern of forced convection and natural circulation in a Calandria vessel. The wall- and corepeaked void distribution of the DOBO test were successfully predicted while the phase interface models need to be improved for a better prediction of the transition region. Porous media models have been validated through the analysis of moderator temperature distribution of the STERN experiment.

Multi-scale applications using the CUPID code have been demonstrated in both explicitly- and implicitlycoupled ways. In the explicitly-coupled calculation, the local boiling heat transfer of a PCCT has been successfully predicted using the results of component-scale analysis. For an implicit-coupled calculation, the CUPID code has been coupled with the system thermal hydraulics code, MARS. The pressure equations of the two codes are solved simultaneously. This is very useful for a fast transient calculation. The ROCOM test has been successfully simulated using the MARS-CUPID code, where the pressure vessel was calculated by the CUPID code and the rest of the system was modeled using the MARS code.

It has been shown, throughout the validation and several assessments, that the CUPID code can be applied to the analysis of two-phase flows of PWRs at both CFD and component scales. In the CFD scale, however, the application is limited to bubbly or mist flows and further development of relevant physical models is required for the application to other flow patterns.

\section{ACKNOWLEDGMENTS}

This work was supported by the 2011 Specialization Project Research Grant funded by the Pusan National University and Nuclear Research \& Development Program of the NRF (National Research Foundation of Korea) grant funded by the MEST (Ministry of Education, Science and Technology) of the Korean government (Grant code: M20702040003-08M0204-00310).

\section{NOMENCLATURE}

$A_{i} \quad$ Interfacial area concentration

$A_{f} \quad$ Face area between cells

$C_{D}$ Drag force coefficient

$C_{\text {lift }}$ Lift force coefficient

$c_{P}$ Specific heat

$D$ Diameter

$e$ Internal energy

$F$ Interfacial force term

$h$ Enthalpy

$H$ Interfacial heat transfer coefficient

$k$ Conductivity

$N$ Number of faces at a cell

$m$ Mass

$M$ Momentum transfer term

$Q \quad$ Interfacial heat transfer term

$\dddot{q} \quad$ Volumetric heat source or sink

Re Reynolds number

$S$ Entrainment, de-entrainment rate

St Stanton number

$\vec{S}_{f} \quad$ Face vector

$T$ Temperature

$\delta t \quad$ Time step

$\vec{u} \quad$ Velocity vector

$V$ Cell volume

$w$ Weighting factor

$X_{n}$ Non-condensable gas quality

\section{Greek Letter}

$\alpha$ Volume fraction

$\beta$ Coefficient of pressure gradient

$\delta \quad$ Variation, characteristic length

$\gamma$ Dimensionless void fraction gradient

$\eta$ Droplet field fraction in liquid phase

$\rho$ Density

$\Gamma \quad$ Bulk phase change rate

$\Omega$ Total phase change rate

$\Psi$ Volume flux

\section{Superscripts}

* Intermediate value

n Old time step

$\mathrm{n}+1$ New time step

$v m$ Virtual mass

\section{Subscripts}

$b$ Bubble

bc Bubble-churn transition

$c$ Continuous phase

cm Churn-mist transition

d Droplet

$D E$ De-entrainment 
E Entrainment

$f \quad$ Cell face, fluid

$F$ Friction

$g$ Gas

$i \quad$ Interface

$k$ Gas, liquid or droplet

$l \quad$ Liquid

$m$ Mixture

$n \quad$ Non-condensable

$\tau$ Tangential

sat Saturation

$s \quad$ Steam

$v \quad$ Vapor

$v m$ Virtual mass

\section{REFERENCES}

[1] M.E. Conner, E. Baglietto and A.M. Elmahdia, "CFD Methodology and Validation for Single-Phase Flow in PWR Fuel Assemblies," Nuclear Engineering and Design, 240, pp. 2088-2095 (2009).

[2] W.K. In, "CFD Simulations of a Flow Mixing and Heat Transfer Enhancement in an Advanced LWR Nuclear Fuel Assembly," Paper 1053, Proc. of the 2007 International Meeting on LWR Fuel Performance, San Francisco, California, USA, September 30-October 3, 2007.

[ 3 ] J.J. Jeong, K.S. Ha, B.D. Chung, and W.J. Lee, "Development of a multi-dimensional thermal-hydraulic system code, MARS 1.3.1," Annals of Nuclear Energy, 26(18), pp. 1611-1642 (1999).

[4] The RELAP5-3D Code Development Team, RELAP5-3D code manual volume I: Code structure, system models and solution methods, Idaho National Engineering and Environmental Laboratory (2001).

[ 5 ] J.J. Jeong, I. Dor, and D. Bestion, "Improvement and assessment of the CATHARE2 three-dimensional module compared with the UPTF downcomer test 7," Nuclear Technology, 117, pp. 267-280 (1997).

[6] J.J. Jeong, H.Y. Yoon, I.K. Park, H.K. Cho, and J. Kim, "A Semi-implicit numerical scheme for transient two-phase flows on unstructured grids," Nuclear Engineering and Design, 238, pp. 3403-3412 (2008).

[7] H.Y. Yoon and J.J. Jeong, "A continuity-Based Semiimplicit Scheme for Transient Two-Phase Flow," J. of Nuclear Science and Technology, 47(9), pp. 779-789 (2010).

[ 8 ] H.Y. Yoon et al., CUPID code Manual Volume I: Mathematical Models and Solution Methods, KAERI/TR-4403 /2011, Korea Atomic Energy Research Institute, 2011.

[9] J.J. Jeong et al., "The CUPID Code Development and Assessment Strategy," Nuclear Engineering and Technology, 42(6), pp. 636-655 (2010).

[10] B.J. Yun, D.J. Euh, C.-H. Song, "Downcomer boiling phenomena during the reflood phase of a large-break LOCA for the APR1400," Nuclear Engineering and Design, 238, pp. 2064-2074 (2008).

[11] R.G. Huget, J.K. Szymanski, and W.I. Midvidy, "Experimental and Numerical Modelling of Combined Forced and Free Convection in a Complex Geometry with Internal Heat Generation," Proc. of $9^{\text {th }}$ International Heat Transfer Conference, 3, 327, 1990.

[12] G. Yadigaroglu, "Computational fluid dynamics for nuclear applications: From CFD to multi-scale CMFD," Nuclear Engineering and Design, 235(2-4), pp. 153-164 (2005).

[13] D. Bestion, "From the Direct Numerical Simulation to System Codes - Perspective for the Multi-scale Analysis of LWR Thermalhydraulics," Nuclear Engineering and Technology, 42(6), pp.609-619 (2010).

[14] K.H. Kang, B.U. Bae, S. Kim, Y.J. Cho, Y.S. Park, B.D. Kim, "Experimental Study on the Operational and the Cooling Performance of the APR+ Passive Auxiliary Feedwater System," Proc. of ICAPP'12, Chicago, USA, June, 2012.

[15] S. Kliem, R. Franz, Quick-look report of the ROCOM Tests 1.1 and 1.2 conducted within the OECD PKL2 Project, Institutsbericht FZD $\backslash F W S \backslash 2010 \backslash 07$.

[16] M. Ishii and T. Hibiki, Thermo-Fluid Dynamics of TwoPhase Flow, Springer (2006).

[17] C. Frepoli, J.H. Mahaffy, and K. Ohkawa, "Notes on the implementation of a fully-implicit numerical scheme for a two-phase three-field flow model," Nuclear Engineering and Design, 225, pp. 191-217 (2003).

[18] A. Tentner, et al., "Computational fluid dynamics modeling of two-phase flow topologies in a boiling water reactor fuel assembly," Proc. of ICONE16, Orlando, USA, 2008.

[19] T. Hibiki, T.H. Lee, J.Y Lee, M. Ishii, "Interfacial area concentration in boiling bubbly flow systems," Chemical Engineering Science 61, pp. 7979-7990 (2006).

[20] I. Kataoka, M. Ishii, K. Mishima, "Generation and Size Distribution of Droplet in Annular Two-Phase Flow," Trans. ASME J. Fluid Engineering 105, pp. 230-238 (1983).

[21] P. Coste, J. Pouvreau, J. Laviéville, M. Boucker, 2008. "Status of a Two-phase CFD Approach to the PTS Issue," Proc. of XCFD4NRS Workshop, Grenoble, France, September, 2008.

[22] M. Ishii, T.C. Chawla, Local drag laws in dispersed twophase flow, Argonne National Lab. Report, ANL-79-105, 1979.

[23] A. Tomiyama, H. Tamia, I. Zun, and S. Hosokawa, "Transverse migration of single bubbles in simple shear flows," Chemical Engineering Science, 57, 1849-1858 (2002).

[24] S.P. Antal, R.T. Lahey, J.E. Flaherty, "Analysis of phase distribution in fully developed laminar bubbly two-phase flow," International Journal of Multiphase Flow, 7, pp. 635-652 (1991).

[25] A.D. Burns, T. Frank, I. Hamill, J.M. Shi, The Favre "Averaged drag model for turbulence dispersion in Eulerian multiphase flow," ICMF'04, Yokohama, Japan, 393, 2004.

[26] D. Drew, L. Cheng, R.T. Lahey, "The analysis of virtual mass effect in two-phase flow," Int. J. Multiphase Flow, 5, pp.233-272 (1979).

[27] W.E. Ranz, W.R. Marshall Jr., "Evaporation from drops," Chemical Engineering Progress, 48, pp.141-144 (1952).

[28] G.I, Hadaller, et al., "Frictional Pressure Drop for Staggered and In Line Tube Bank with Large Pitch to Diameter Ratio," Proceedings of 17th CNS Conference, Federiction, New Brunswick, Canada, June 9-12, 1996.C.

[29] Yoon, et al., "Development and Validation of the 3-D Computational Fluid Dynamics Model for CANDU-6 Moderator Temperature Predictions", Nuclear Technology, 148, pp.259-267 (2004).

[30] B.U. Bae, B.J. Yun, S. Kim, K.H. Kang, "Design of condensation heat exchanger for the PAFS (Passive Auxiliary 
Feedwater System) of APR+ (Advanced Power Reactor Plus)," Annals of Nuclear Energy, 46, pp.134-143 (2012).

[31] S.Y. Lee, J.J. Jeong, S.H. Kim, and S.H. Chang, "COBRA
/RELAP5; A merged version of the COBRA-TF and RELAP5/MOD3 codes," Nuclear Technology, 99, 177187 (1992). 